\title{
Competência Moral e Espiritualidade na Educação Médica: Realidade ou Desafio?
}

\author{
Moral Competence and Spirituality in Medical \\ Education: Challenge or Reality?
}

\author{
Natália Wolmer de Melo \\ Edvaldo Souza \\ Leopoldo Barbosa ${ }^{I I}$
}

\section{PALAVRAS-CHAVE \\ - Moral; \\ - Espiritualidade; \\ - Estudantes de Medicina; \\ - Educação Médica.}

\section{KEYWORDS}

- Moral;

- Spirituality;

- Medical Students;

- Medical Education.
REVISTA BRASILEIRA DE EDUCAÇÃO MÉDICA
${ }^{I}$ Faculdade Pernambucana de Saúde, Recife, PE, Brasil.

"Universidade Federal de Pernambuco, Recife, PE, Brasil.

\begin{abstract}
RESUMO
Estudantes de Medicina iniciam a faculdade idealistas, mas muitos consideram sair indiferentes: este éo maior desafio da educação médica. Estudos ratificam uma involução da competência moral durante o curso. Outro interesse crescente no meio acadêmico é a espiritualidade. Objetivo: Avaliar a relação entre competência moral e espiritualidade dos estudantes de Medicina. Métodos: Estudo descritivo transversal, com 121 estudantes. A coleta foi realizada por meio do software limesurvey, contendo o TCLE e três questionários: sociodemográfico, teste de competência moral de George Lind, calculado em planilha do Excel, e escala de espiritualidade de Pinto e Pais-Ribeiro. Utilizou-se o software Stata 12.0 na análise. Resultados: Ter religião está associado a maior espiritualidade. Em relação ao gênero feminino e a ter pais médicos, houve tendência de associação com espiritualidade elevada. O escore $C$ manteve-se crescente durante o curso. Na relação entre espiritualidade e competência moral, estudantes com baixa espiritualidade apresentaram tendência a escore $C$ maior. Conclusão: Estudantes mais espiritualizados apresentam tendência a ter competência moral menor. O questionário de espiritualidade, contudo, está intrincado com dimensões religiosas, que tendem a limitar o desenvolvimento da competência moral na população estudada.
\end{abstract}

Introduction: Medical undergraduates start college as idealists, but many are considered to leave indifferent: this is the biggest challenge in medical education. Studies have indicated a regression in moral competence throughout the undergraduate course. Another matter of growing interest in the academic sphere is spirituality. Objective: To evaluate the relationship between moral competence and spirituality among medical students. Methods: Descriptive cross-section study of 121 students. Data were collected using Limesurvey software, with the Informed Consent Form and three questionnaires: sociodemographic, George Lind's moral competence test, calculated in Excel, and Pinto e Pais-Ribeiro's spirituality scale. Stata 12.0 was used for the analysis. Results: Having a religion is associated to a higher degree of spirituality. As regards gender and doctor parents, there was an associative trend with heightened spirituality. The $C$ score continued to increase throughout the course. In terms of the relationship between spirituality and moral competence, relatively unspiritual students presented a higher $\mathrm{C}$ score. Conclusion: Highly spiritual students showed a tendency toward a lower moral competence. The spirituality questionnaire, however, is pervaded by religious dimensions, which tend to limit the development of moral competence in the studied population. 


\section{INTRODUÇÃO}

Por questões políticas, sociais e científicas, a educação moral e a moralidade vêm despertando acentuado interesse no meio acadêmico ${ }^{1}$, uma vez que eventos como a ameaça do terrorismo, crises econômicas globais, crescimento do crime entre os jovens, gravidez na adolescência e suicídio reacenderam o interesse pela ética e educação moral, e, entre várias outras, são situações que levantam a hipótese de crise moral na sociedade atual ${ }^{2,3}$.

Inicialmente, a construção moral foi estabelecida pelo pensamento e sentimento religioso, quando se recorria à concepção de um ser ou seres divinos a quem se devia a vontade da ação justa e certa4. Mas é com Aristóteles que o conceito adquire importância central para a reflexão filosófica ${ }^{4,5}$. A ética passa a se destinar à compreensão dos critérios e valores que orientam o julgamento da ação humana como conduta moralmente errada ou correta e passa a ser um sistema de ação ${ }^{4}$.

Outras escolas filosóficas que abordam a questão moral sucederam esses filósofos, passando pela filosofia medieval e pela idade moderna, chegando à idade contemporânea, quando entram em cena Piaget, pioneiro do ponto de vista cognitivista da moralidade, e Kohlberg ${ }^{6}$, que complementou o trabalho de Piaget e lançou as bases para o atual debate na psicologia sobre o desenvolvimento moral ${ }^{1}$.

Segundo Piaget, o ser humano não é uma tábula rasa, em que se inscrevem os valores sociais e morais esperados pelos adultos, professores ou autoridades. Em contextos interpessoais e de ensino-aprendizagem, por meio de vivência e exemplos, o ser humano desenvolve a capacidade de pensar sobre si e os demais.

Conforme Kohlberg, competência moral é "a capacidade de tomar decisões e emitir juízos morais (baseados em princípios internos) e agir de acordo com tais juízos"7,8. Para o desenvolvimento desta competência, existe uma sequência universal e invariante de seis estágios ${ }^{9}$. Para mensurar a competência moral, Kohlberg propôs uma forma de avaliação e mensuração: a entrevista de juízo moral (Moral Judgment Interview - MJI). Desde então, diversos estudiosos buscaram desenvolver novos instrumentos mais breves de avaliação do juízo moral ${ }^{8}$.

George Lind, da Universidade de Konstanz, desenvolveu o Moral Judgement Test (MJT) ou teste do julgamento moral. Segundo Lind, essas habilidades e competências morais podem e devem ser construídas ao longo da vida, isto é, infância, juventude e idade adulta ${ }^{10}$.

No caso de estudantes universitários, anima o educador o fato de ser possível resgatar, estimular e aprimorar conteúdos e princípios latentes, favorecendo a formação de um pro- fissional digno, que reúna conhecimento técnico primoroso, sem deixar de ser depositário das aspirações humanistas que orientam tanto os princípios bioéticos quanto os direitos humanos $^{4}$.

No curso médico, embora a medicina endosse um código de ética e encoraje um alto nível de caráter moral entre os médicos ${ }^{11}$, considera-se que estudantes de Medicina iniciam a faculdade como idealistas, mas muitos deles sentem que saem da faculdade frios e indiferentes. Este é o maior desafio da educação médica, pois frequentemente o desenvolvimento moral fica estancado ou há regressão ${ }^{12}$.

Diversas pesquisas, inclusive na realidade brasileira, utilizando diferentes instrumentos, corroboram tal afirmação ${ }^{13,14,15}$. Um estudo longitudinal na Alemanha que usou o teste de juízo moral de Lind encontrou que os estudantes de Medicina mostram regressão na competência do julgamento moral ( $C$ -score), enquanto outros estudantes universitários em geral mostram aumento notável. Esse estudo também sugere que a regressão no julgamento moral deve estar relacionada ao ambiente de aprendizado médico ${ }^{14}$.

A medicina moderna, entretanto, busca caminhos para o tratamento mais integral dos pacientes, deixando de seguir o modelo biológico exclusivo para ser complementada pelos modelos psicológico, social, ecológico e espiritual ${ }^{16}$.

A espiritualidade, por sua vez, vem sendo cada vez mais estudada no meio acadêmico. A Associação Americana de Faculdades Médicas (AAMC), a Organização Mundial de Saúde (OMS) e a Comissão Conjunta de Acreditação de Organizações de Saúde (JCAHO) também recomendam abordar questões espirituais no atendimento clínico e na educação dos profissionais de saúde. Isto porque estudos mostram associação entre saúde física e mental e crenças espirituais e religiosas, adesão ao tratamento, tomada de decisões médicas, questões éticas e morais, e até mesmo de sobrevivência ${ }^{17}$.

Embora não exista uma definição única para espiritualidade, esta se refere a práticas não necessariamente ligadas às religiões e ressalta principalmente a dinâmica de aproximação com o eu profundo ${ }^{18}$.

Um estudo que analisou as crenças de estudantes de Medicina sobre a relação entre espiritualidade e saúde e o nível de espiritualidade que o currículo deve conter mostrou que os resultados do questionário de 254 estudantes de Medicina indicam que a religiosidade e a espiritualidade são importantes, sendo a segunda mais importante do que a primeira ${ }^{19}$.

Outro estudo que avalia a implementação de curso eletivo em medicina e espiritualidade incluiu como pontos fortes relatados sobre o curso, entre outros, princípios universais e oportunidade para autorreflexão ${ }^{20}$. 
Considera-se também que a formação humanística implica a necessidade do autoconhecimento e envolve a consciência do outro e de seus valores ${ }^{21}$.

Entretanto, poucos estudos relacionam ética e moral de estudantes de Medicina com espiritualidade, e até o presente momento não foram encontrados estudos que avaliem a relação entre competência moral e espiritualidade.

Então, questiona-se se a espiritualidade, enquanto dimensão humana ${ }^{16}$, também estaria envolvida na construção, modificação e consolidação do juízo moral dos estudantes de Medicina.

\section{MÉTODO}

Este estudo é descritivo, de corte transversal, no qual os estudantes de Medicina da Faculdade Pernambucana de Saúde (FPS) - instituição com metodologia ativa, módulos transversais de ética e módulo de teologia - foram caracterizados sociodemograficamente e avaliados quanto à competência do julgamento moral e à espiritualidade. Todos os estudantes matriculados no ano de 2014 no curso médico foram convidados, e a coleta de dados ocorreu de agosto a setembro de 2014 .

A coleta de dados foi iniciada após a aprovação do Comitê de Ética em Pesquisa da FPS e foi realizada por e-mail, através do software limesurvey, contendo o TCLE - pré-requisito para prosseguir com o preenchimento dos questionários - e três questionários breves: questionário sociodemográfico, teste de competência moral de George Lind e escala de espiritualidade de Pinto e Pais-Ribeiro.

O questionário sociodemográfico foi construído alinhado aos achados observados e relatados em outros estudos e contém informações para caracterizar a população de estudo quanto a gênero, idade, se viveu ou não a maior parte de sua vida em região metropolitana, ano do curso em graduação, religião, onde concluiu o segundo grau (ensino público ou privado), quantas vezes prestou vestibular de Medicina, se possui pais médicos e a forma como a graduação é custeada.

O teste do julgamento moral utilizado é validado em português ${ }^{22}$. É composto por dois dilemas morais e por seis argumentos a favor da decisão do protagonista e seis contrários, e os entrevistados irão opinar numa escala entre 4 (aceita completamente) e -4 (rejeita completamente). Com base no padrão das respostas, gera-se um escore (C-score) correspondente ao grau de competência moral.

A escala de espiritualidade de Pinto e Pais-Ribeiro ${ }^{16}$ é uma escala brasileira, composta por cinco itens centrados em duas dimensões: a dimensão vertical (crença) e a dimensão horizontal (esperança/otimismo). As respostas são dadas em escala Likert composta, com quatro opções: "não concordo", "concordo um pouco", "concordo bastante" e "plenamente de acordo". O ponto médio é de 2,5. Valores inferiores a esse ponto de corte correspondem a escores baixos, e valores superiores, a escores elevados.

$\mathrm{Na}$ análise do escore C foi utilizada planilha do Excel, conforme orientação do autor, e na análise geral dos dados foi usado o software Stata, versão 12.0 .

As características da população em estudo foram apresentadas por meio de distribuições de frequência e representadas por média e desvio padrão quando a variável era do tipo quantitativa. Na análise da associação das variáveis estudadas se aplicou o teste Qui-Quadrado de Pearson nas comparações com as variáveis categóricas. No caso da associação com a idade, foi aplicada uma comparação entre médias pelo teste $t$ de Student.

Testando a proposição de normalidade pelo teste de Komogorov do escore $C$ do MJT, a distribuição do escore não foi normalmente distribuída ( $\mathrm{p}=0,0002)$. Assim, a estatística descritiva na análise comparativa do escore foi apresentada pela mediana e pelo intervalo interquartílico, e o teste aplicado foi o não paramétrico de Mann-Whitney na comparação com os grupos segundo a classificação da espiritualidade. A significância adotada no teste foi de $5 \%$ ( $p<0,05)$. A fim de ajustar as associações observadas na análise univariada, foi realizada uma análise multivariada, aplicando-se um modelo de regressão logística, com ponto de corte de entrada no modelo de $20 \%(p<0,2)$.

\section{RESULTADOS}

Dos 678 estudantes convidados, participaram do estudo 126 alunos de Medicina da FPS, mas, para fins do estudo, foi necessário excluir cinco deles, pois suas respostas ao questionário MJT foram inviabilizadas para o cálculo do escore C. Logo, o total da amostra foi de 121 estudantes. Entre estes, 71,1\% corresponderam ao gênero feminino. A média de idade do grupo estudado foi 22,5 anos, variando entre 17 e 37 anos. Em relação ao ciclo do curso médico, 21,5\% corresponderam ao primeiro ano do curso, $42,2 \%$ ao segundo ano, $9,9 \%$ ao terceiro ano, $16,5 \%$ ao quarto ano, e 9,9\% aos dois últimos anos.

Quanto à religião, 89,3\% afirmaram ter alguma religião, sendo $52,1 \%$ católicos. Quanto ao local em que viveu a maior parte de sua vida, 80,2\% referiram ter vivido em região metropolitana.

Dos estudantes, 92\% referiram ter cursado o ensino médio em escola privada. E $25 \%$ realizaram apenas uma vez o vestibular, enquanto $30,6 \%$ fizeram dois vestibulares, $26,4 \%$ fizeram três tentativas e $18,2 \%$ prestaram quatro ou mais vestibulares. Entre os estudantes, $81 \%$ têm um dos pais médico. Em relação ao custeio da faculdade, $47,9 \%$ referiram ter seu curso custeado pelos pais, e, em segundo lugar, 25,6\% dos estudantes têm o curso custeado pelo Fies (Tabela 1). 


\begin{tabular}{|c|c|}
\hline \multicolumn{2}{|c|}{$\begin{array}{c}\text { TABELA } 1 . \\
\text { Características da população de estudo: } \\
\text { estudantes de Medicina de instituição de educação } \\
\text { superior da cidade do Recife, em } 2014\end{array}$} \\
\hline Características & Estatísticas \\
\hline \multicolumn{2}{|l|}{ Sexo } \\
\hline Feminino & $86(71,1 \%)$ \\
\hline Masculino & $35(28,9 \%)$ \\
\hline \multicolumn{2}{|l|}{ Idade } \\
\hline Média \pm dp (minimo; máximo) & $22,5 \pm 3,2(17 ; 37)$ \\
\hline \multicolumn{2}{|l|}{ Local de residência na RMR } \\
\hline Sim & $97(80,2 \%)$ \\
\hline Não & $24(19,8 \%)$ \\
\hline \multicolumn{2}{|l|}{ Religião } \\
\hline Agnóstico/Ateu & $13(10,7 \%)$ \\
\hline Católico & $63(52,1 \%)$ \\
\hline Espírita & $19(15,7 \%)$ \\
\hline Evangélica & $17(14,1 \%)$ \\
\hline Outras $^{\mathrm{a}}$ & $9(7,4 \%)$ \\
\hline \multicolumn{2}{|c|}{ Relacionadas à graduação em Medicina } \\
\hline \multicolumn{2}{|c|}{ Período } \\
\hline Primeiro & $26(21,5 \%)$ \\
\hline Terceiro & $51(42,2 \%)$ \\
\hline Quinto & $12(9,9 \%)$ \\
\hline Sétimo & $20(16,5 \%)$ \\
\hline Nono & $7(5,8 \%)$ \\
\hline Décimo primeiro & $5(4,1 \%)$ \\
\hline \multicolumn{2}{|c|}{ Escola em que concluiu ensino médio } \\
\hline Pública & $10(8,3 \%)$ \\
\hline Privada & $111(91,7 \%)$ \\
\hline \multicolumn{2}{|c|}{$\begin{array}{l}\text { Número de vezes em que prestou vestibular } \\
\text { para Medicina }\end{array}$} \\
\hline Uma vez & $30(24,8 \%)$ \\
\hline Duas vezes & $37(30,6 \%)$ \\
\hline Três vezes & $32(26,4 \%)$ \\
\hline Quatro vezes ou mais & $22(18,2 \%)$ \\
\hline \multicolumn{2}{|l|}{ Possui pais médicos } \\
\hline Não & $23(19,0 \%)$ \\
\hline Sim, pai ou mãe & $90(74,4 \%)$ \\
\hline Sim, ambos & $8(6,6 \%)$ \\
\hline \multicolumn{2}{|l|}{ Custeio do curso de Medicina } \\
\hline Pais & $58(47,9 \%)$ \\
\hline Prouni & $14(11,6 \%)$ \\
\hline Fies & $31(25,6 \%)$ \\
\hline Pais e Fies & $16(13,2 \%)$ \\
\hline Outros & $2(1,7 \%)$ \\
\hline
\end{tabular}

${ }^{a}$ Outras: budista (2), judaica (6); outras (1).
Quanto à avaliação da espiritualidade, mensurada pela escala de Pinto e Pais-Ribeiro em alta e baixa, foi encontrado que $14 \%(17 / 121)$ foram classificados com baixa espiritualidade.

O escore C da escala MJT da amostra apresentou distribuição não normal, tendo como valor mínimo 0 e máximo 43,2. A mediana foi 10,8 , com intervalo interquartílico de 5,3 e 17,6 (percentil 25 e percentil 75). Quando correlacionados os aspectos sociodemográficos com o grau de espiritualidade, houve associação estatisticamente significante $(p<0,05)$ quanto a gênero, condição de ter religião e ter pais médicos (Tabela 2).

Quanto ao gênero, estudantes do sexo masculino apresentaram maior percentual de baixa espiritualidade $(28,6 \%)$ quando comparados às do sexo feminino $(8,1 \%)$. Quanto à condição de ter religião, a frequência de baixa espiritualidade foi aproximadamente seis vezes maior em quem se declarou agnóstico ou ateu, quando comparados aos estudantes que afirmaram ter alguma religião. Em relação à condição de ter pais médicos, $89,9 \%$ dos estudantes que têm um dos pais médico possuem espiritualidade elevada.

Contudo, após análise multivariada, o gênero, a condição de ter pais médicos e o escore de moralidade não apresentaram significância estatística. No entanto, vale salientar que as associações obtidas após o ajuste foram limítrofes, mostrando uma tendência de associação a partir de um tamanho maior de amostra.

Todavia, quanto à condição de ter religião, 90,7\% dos estudantes que têm religião obtiveram grau de espiritualidade elevado, e, mesmo após a análise multivariada, a associação foi mantida (Tabela 2).

Em relação ao escore $C$, seu valor médio no grupo avaliado foi de 12,7, com desvio padrão igual a 9,3. Quando relacionado esse escore com os aspectos sociodemográficos, só houve relação estatisticamente significativa entre os períodos de graduação do curso médico, uma vez que o escore $C$ foi crescente ao longo dos anos (Tabela 3).

No que se refere à associação do grau de espiritualidade com a moralidade, estudantes classificados com baixa espiritualidade apresentaram maior escore no MJT, com escore mediano de 15,7 pontos para os estudantes classificados com baixa espiritualidade e de 9,9 pontos entre aqueles que têm espiritualidade elevada, mostrando tendência de associação de estudantes menos espiritualizados com maior escore de competência moral, após análise multivariada (Tabela 2).

\section{DISCUSSÃO}

O presente estudo avaliou a relação entre competência moral e grau de espiritualidade de estudantes de Medicina da Facul- 
TABELA 2.

Associação dos fatores sociodemográficos relacionados à graduação de Medicina e escore C do MJT entre os estudantes de Medicina de uma instituição de educação superior privada do Recife em 2014

\begin{tabular}{|c|c|c|c|c|}
\hline \multirow{2}{*}{ Características } & \multicolumn{2}{|c|}{ Escala de espiritualidade } & \multirow{2}{*}{ p-valor } & \multirow{2}{*}{ p-valor ajustado } \\
\hline & Baixa & Elevada & & \\
\hline \multicolumn{5}{|c|}{ Sociodemográficas } \\
\hline Feminino & $7(8,1 \%)$ & $79(91,9 \%)$ & $0,003^{+}$ & 0,061 \\
\hline Masculino & $10(28,6 \%)$ & $25(71,4 \%)$ & & \\
\hline \multicolumn{5}{|l|}{ Idade } \\
\hline \multicolumn{5}{|l|}{ Local de residência na RMR } \\
\hline Não & $1(4,2 \%)$ & $23(95,8 \%)$ & 0,120 & - \\
\hline Sim & $16(16,5 \%)$ & $81(83,5 \%)$ & & \\
\hline \multicolumn{5}{|l|}{ Tem religião } \\
\hline Sim & $10(9,3 \%)$ & $98(90,7 \%)$ & $0,000^{+}$ & $0,011^{+}$ \\
\hline Não & $7(53,8 \%)$ & $6(46,2 \%)$ & & \\
\hline Quinto e sétimo & $5(15,6 \%)$ & $27(74,4 \%)$ & & \\
\hline Nono e décimo primeiro & $2(16,7 \%)$ & $10(83,3 \%)$ & & \\
\hline \multicolumn{5}{|l|}{ Possui pais médicos } \\
\hline Sim & $10(10,2 \%)$ & $88(89,8 \%)$ & $0,012^{+}$ & 0,082 \\
\hline Não & $7(30,4 \%)$ & $16(69,6 \%)$ & & \\
\hline \multicolumn{5}{|l|}{ Número de vestibulares } \\
\hline Um & $5(16,7 \%)$ & $25(83,3 \%)$ & 0,705 & - \\
\hline Dois & $6(16,2 \%)$ & $31(83,8 \%)$ & & \\
\hline Três ou mais & $6(11,1 \%)$ & $48(88,9 \%)$ & & \\
\hline \multicolumn{5}{|l|}{ Custeio do curso de Medicina } \\
\hline Pais & $10(17,2 \%)$ & $48(82,8 \%)$ & 0,780 & - \\
\hline
\end{tabular}

${ }^{+}$Associação estatisticamente significante $(p<0,05)$.

a Associação com ajuste da análise multivariada (regressão logística).

\section{Tabela 3.}

Associação dos fatores sociodemográficos relacionados à graduação de Medicina e escore C do MJT entre os estudantes de Medicina de uma instituição privada do Recife em 2014

$\begin{array}{lll}\text { Características sociodemográficas } & \text { Escore } C \text { do } M T J & \text { p-valor }\end{array}$

Sexo

$\begin{array}{ll}\text { Feminino } & 10,86(6,86 ; 18,35) \\ & 10,06(4,68 ; 17,62)\end{array}$

$10,06(4,68 ; 17,62)$

0,801

Idade

Coef. correlação

$-0,007$

0,938

Local de residência na RMR

Não

$9,11(4,23 ; 16,91)$

Sim 


\begin{tabular}{|c|c|c|}
\hline \multicolumn{3}{|c|}{$\begin{array}{l}\text { TABELA } 3 . \\
\text { S relacionados à graduação de Medicina e escore C do } \\
\text { na de uma instituição privada do Recife em } 2014\end{array}$} \\
\hline Características sociodemográficas & $\begin{array}{l}\text { Escore } C \text { do } M T J \\
\text { Mediana }\left(\mathbf{P}_{25} ; \mathbf{P}_{75}\right)\end{array}$ & p-valor \\
\hline \multicolumn{3}{|l|}{ Tem religião } \\
\hline Sim & $10,19(5,21 ; 17,28)$ & \multirow{2}{*}{0,120} \\
\hline Não & $15,96(6,16 ; 22,86)$ & \\
\hline \multicolumn{3}{|c|}{ Relacionadas à graduação em Medicina } \\
\hline \multicolumn{3}{|l|}{ Período } \\
\hline Primeiro e terceiro & $9,98(5,26 ; 16,27)$ & \multirow{3}{*}{$0,010^{+}$} \\
\hline Quinto e sétimo & $10,34(4,66 ; 15,71)$ & \\
\hline Nono e décimo primeiro & $29,43(10,1 ; 33,11)$ & \\
\hline \multicolumn{3}{|l|}{ Possui pais médicos } \\
\hline Sim & $10,24(4,78 ; 16,97)$ & \multirow{2}{*}{0,556} \\
\hline Não & $11,11(6,16 ; 20,02)$ & \\
\hline \multicolumn{3}{|l|}{ Número de vestibulares } \\
\hline Um & $9,91(5,26 ; 18,63)$ & \multirow{3}{*}{0,714} \\
\hline Dois & $11,02(7,73 ; 15,97)$ & \\
\hline Três ou mais & $9,93(4,08 ; 17,62)$ & \\
\hline \multicolumn{3}{|l|}{ Custeio do curso de Medicina } \\
\hline Pais & $11,52(5,26 ; 19,45)$ & \multirow{4}{*}{0,170} \\
\hline Prouni & $12,08(2,33 ; 23,24)$ & \\
\hline Fies & $8,71(2,81 ; 14,28)$ & \\
\hline Pais e Fies & $13,74(8,09 ; 19,04)$ & \\
\hline
\end{tabular}

${ }^{+}$Associação estatisticamente significante $(p<0,05)$.

dade Pernambucana de Saúde. Além disso, correlacionou os aspectos sociodemográficos - gênero, idade, local onde viveu a maior parte de sua vida, religião, ano da graduação, onde concluiu o segundo grau, número de vestibulares que prestou para Medicina, se tem pais médicos, forma como a graduação é custeada - com o grau de espiritualidade e com o escore C (escore de competência moral) desses estudantes.

Realizou-se a pesquisa online com o intuito de ser mais conveniente para o respondente, no tempo e local de cada um, o que aumentaria a chance de as respostas serem mais fidedignas, pois, entre outras vantagens, o pesquisador teria alto controle sobre o preenchimento da pesquisa ${ }^{23}$. Foram realizadas visitas aos estudantes antes das tutorias para convidá-los a participar da pesquisa e para prestar esclarecimentos sobre o estudo.

Contudo, obtivemos perda maior que a esperada, pois, conforme a literatura, questionários enviados a entrevistados alcançam, em média, 25\% de devolução ${ }^{24}$, e neste estudo apenas $18,5 \%$ da população responderam de forma completa e adequada ao questionário, tendo sido esta uma das limitações do estudo. Acreditamos que alguns fatores tenham contribu- ído para essa redução na participação dos estudantes: o fato de diversas pesquisas estarem ocorrendo ao mesmo tempo na instituição, a existência de poucas pesquisas online na FPS e a própria rotina com muitas atividades dos estudantes, em especial daqueles dos últimos dois anos do curso.

Neste grupo estudado, composto em sua maioria pelo gênero feminino, no início da vida adulta, com maioria pertencente a classes econômicas mais favorecidas, tendo pais médicos e a maioria tendo alguma religião, foi encontrada tendência, ou seja, resultados limítrofes. Isto indica que a associação possivelmente ocorreria com um $\mathrm{N}$ maior do que os estudantes que obtiveram baixa espiritualidade, ou seja, pontuaram abaixo de 2,5 pontos na escala de espiritualidade de Pinto-Paes Ribeiro ${ }^{23}$ e alcançaram maiores índices do escore C, que avalia a competência moral. Estes estudantes obtiveram o escore C mediano de 15,7. Já os estudantes com espiritualidade elevada obtiveram escore $C$ mediano de 9,9 , o que, curiosamente, está de acordo com a literatura.

A escala escolhida para avaliar a espiritualidade foi a escala de espiritualidade de Pinto-Paes Ribeiro ${ }^{16}$, escala simples, objetiva e de fácil aplicação, além de ter sido criada original- 
mente em português. Entretanto, vale ressaltar que não existe uma escala padrão ouro para avaliar espiritualidade ${ }^{25}$, assim como a maioria das escalas que buscam avaliar esse constructo na língua portuguesa não foi completamente validada, mas tem boas qualidades psicométricas ${ }^{26}$.

Nesta escala existem duas dimensões de avaliação da espiritualidade: uma vertical, relacionada a crenças, e uma dimensão horizontal, associada a esperança/otimismo. A primeira e a segunda questão da escala correspondem à dimensão vertical, e 13,2\% e 10,7\%, respectivamente, não estavam de acordo com essas questões. Dessa forma, essas duas questões, que avaliam as crenças - e, portanto, está aí presente o aspecto religioso -, contribuíram de forma importante para o baixo grau de espiritualidade de $14 \%$ dos respondentes.

Assim, ao se analisarem estudos que comparam competência moral com religiosidade, uma vez que até o momento não foram encontrados artigos que avaliem espiritualidade e competência moral, observa-se que indivíduos com posturas mais religiosas, mais dogmáticas, têm competência moral menos desenvolvida, ao passo que indivíduos menos dogmáticos tendem a elevar sua competência moral, corroborando o achado acima ${ }^{27,28,29}$. No presente estudo, os estudantes com baixa espiritualidade (com destaque para não concordância com as questões da dimensão vertical, portanto estudantes provavelmente menos religiosos) obtiveram maior escore de competência moral (mesmo após o ajuste, por análise multivariada de variância, a tendência se manteve).

Ratificando este achado, um estudo no Paquistão evidenciou que estudantes com religiosidade menos dogmática tiveram a competência moral discretamente maior quando comparados a estudantes dogmáticos ${ }^{27}$. Um estudo realizado no Irã também mostrou que a religiosidade dogmática dificulta o desenvolvimento do julgamento e competência moral ${ }^{28}$. No Brasil, Bataglia encontrou que pessoas que se disseram "pouco religiosas" mostraram competência moral discretamente superior em relação aos que se julgaram "muito religiosos" ou "sem religiosidade alguma". Isto sugere, segundo o autor, que pessoas que não seguem estritamente uma direção têm maior flexibilidade de pensamento, o que contribui para maiores escores de competência moral ${ }^{29,30}$.

Outro estudo, que compara estudantes universitários de Psicologia do Brasil e da Alemanha, encontrou que o escore médio de competência moral entre os estudantes alemães é superior ao dos brasileiros. Este fato é embasado pela hipótese de Lind, quando traz que os assuntos orientados pela religião suprimem os aspectos autônomos dos julgamentos morais, quando os dilemas são fortemente defendidos pela Igreja. E o Brasil ainda se encontra na atualidade sob forte influência da religião católica ${ }^{31}$. Este aspecto também pôde ser ressaltado no presente estudo, uma vez que $89,2 \%$ dos estudantes declararam ter religião, e, desses estudantes, 90,7\% obtiveram grau elevado de espiritualidade estatisticamente significativo, sendo a religião mais frequente a católica, correspondendo a $52,1 \%$.

Todavia, outro estudo, de Bataglia e Schillinger-Agati com estudantes universitários com influências religiosas e sem influências, no Brasil, sugeriu que não seria a religião per se, mas, sim, os aspectos culturais do povo brasileiro que poderiam ser responsáveis pelo fenômeno da segmentação moral (valor de escore $\mathrm{C}$ médio diferente de um dilema para o outro na mesma amostra $)^{29}$, o que não foi avaliado neste estudo.

Dessa forma, é possível concluir que, provavelmente, os estudantes que obtiveram menor pontuação na escala de espiritualidade (17 estudantes) conseguiram atingir maiores escores de competência moral porque conseguem se distanciar de aspectos religiosos/culturais de maneira mais efetiva durante a resolução de dilemas morais, no teste de competência moral e, possivelmente, na vida. Porém, os escores de competência moral alcançados pelos estudantes avaliados foram baixos quando comparados com os de outros países e com estudos do Brasil. No presente estudo, o escore C médio geral dos estudantes avaliados foi 12,7.

Uma pesquisa realizada na China em 2011 encontrou escore médio de $31,4^{27}$. Já em estudo realizado no Irã, o escore C foi até $20^{28}$. Na Alemanha, o escore médio ficou em torno de $40^{30}$, e nos EUA foi $23,8^{27}$. Em um estudo realizado em outra faculdade de Medicina do Nordeste brasileiro, foi encontrado escore C médio de 20,5 para o primeiro semestre e de 26,2 para o oitavo semestre ${ }^{31}$. Um estudo posterior, com o mesmo pesquisador deste último estudo, comparou estudantes de Medicina brasileiros e portugueses, e evidenciou que os alunos portugueses tenderam a apresentar escore $C$ mais elevado do que os brasileiros ${ }^{15}$. Schillinger-Agati e Lind evidenciaram escore $C$ de 22,8 para estudantes de universidades mais concorridas e de 13,4 para estudantes de universidades menos concorridas $^{30}$.

Este fato poderia ser semelhante nos estudantes deste estudo, uma vez que são de instituição privada, que costumava ter vestibular menos concorrido que outras faculdades mais tradicionais da cidade, associado aos aspectos culturais e religiosos inerentes da população em questão, como indicado acima.

Contudo, outro aspecto relativo a este grupo de estudantes chama atenção. Diferentemente de diversos estu$\operatorname{dos}^{11,12,14,15,31,32,33}$ que mostraram diminuição ou estagnação da competência moral com o avançar dos períodos de graduação, 
o grupo estudado mostrou movimento inverso, ou seja, estudantes do primeiro e segundo ano tiveram escore $C$ menor que os estudantes dos anos seguintes ( $p<0,010)$.

Possivelmente, a educação médica fornecida teria influenciado esses resultados. A instituição em questão utiliza metodologia ativa, aprendizagem baseada em problemas, com currículo construído em espiral, contendo módulo transversal de ética do primeiro ao sexto ano. Inclui ainda módulo que aborda as diversas características religiosas/culturais do Brasil, além de abrir espaços para os estudantes criarem grupos de atividades variadas, como cineclube e um grupo de estudos em saúde e espiritualidade não vinculado à grade curricular da instituição, mas que funciona há quatro anos regularmente e utiliza o espaço físico da instituição e seus meios de divulgação ${ }^{34,35}$.

Tais características da educação médica oferecida pela instituição possivelmente teriam contribuído para os resultados encontrados nesse grupo. Isto é corroborado pela afirmação de que alta qualidade de educação, com possibilidade de maior tomada de responsabilidade e oportunidade de reflexão com auxílio, leva a efeitos positivos sobre os alunos dogmáticos e não dogmáticos, ao passo que alta religiosidade dogmática parecia neutralizar, se não diminuir, o nível de julgamento moral, mesmo quando foi ofertada educação de alta qualidade ${ }^{28}$.

Tal conclusão é ratificada por um estudo de Schillinger ${ }^{30}$, que comparou 618 estudantes brasileiros com 531 estudantes de dois países de língua alemã (Alemanha e Suíça) do primeiro e do último ano dos cursos de Psicologia, Administração e Medicina. Schillinger encontrou que entre os estudantes de Medicina houve queda no escore C no Brasil e estagnação entre os estudantes de língua germânica. Quanto aos alunos de Administração, houve crescimento no escore entre os de língua germânica e queda entre os brasileiros. E nos estudantes de Psicologia, no Brasil houve crescimento importante entre estudantes de universidades concorridas e queda nos estudantes de universidades menos concorridas, enquanto nos estudantes de língua alemã houve discreto aumento do primeiro ano em relação ao último. $\mathrm{O}$ que a autora sugeriu como possível explicação foi que os estudantes de Psicologia de língua germânica foram expostos desde o começo do curso a role-taking e guided reflection (tomada de responsabilidade e oportunidade de reflexão) ${ }^{30}$. Isto reforça a hipótese de que a competência moral progride à medida que é favorecida por formas institucionalizadas de educação, diferindo do que pregavam Piaget e Kohlberg, e pode haver, sim, uma regressão da competência moral se ela não for estimulada ${ }^{30,31}$.

Lind valida essa hipótese ao afirmar que as habilidades morais ou competências não são inatas nem podem ser ensinadas por meio de palestras simples, mas que o aluno pode (e deve) ser educado ao longo do ciclo de vida, isto é, desde a primeira infância, passando pela infância, juventude e idade adulta ${ }^{10}$. E na instituição do presente estudo a metodologia favorece constantes oportunidades de role-taking e guided reflection nos grupos tutoriais, nas atividades de laboratórios, embasadas na própria metodologia problematizadora e no estímulo à iniciativa de novas atividades para discussão com o grupo de saúde e espiritualidade. Este fato pode ter favorecido a não regressão do escore $C$ com o avançar do curso médico.

Todavia, a participação dos estudantes dos dois últimos anos foi de 9,9\%, e, como relatado em estudo recente, entre estudantes de Medicina a principal queda da competência moral acontece nos anos de prática clínica, provavelmente devido à organização hierárquica da prática clínica, à natureza específica de dilemas morais enfrentados pelos estudantes de Medicina e ao curriculum médico oculto ${ }^{32}$.

Dessa forma, é preciso fazer novos estudos com maior número de estudantes e com maior participação de alunos dos dois últimos anos, no internato, onde existe maior imersão na prática clínica. Também é necessário realizar estudos para compreender por que o escore C está abaixo dos de outros estudos, apesar de não involuir ao longo dos períodos.

Quanto à espiritualidade, embora se saiba que é uma dimensão humana e existam estudos crescentes nesta área, inclusive relacionando espiritualidade e saúde ${ }^{16}$, o maior desafio talvez seja, como reforçam Luchetti e colaboradores ${ }^{25}$, a falta de consenso entre os estudos que avaliam espiritualidade e religiosidade, por serem temas complexos e que envolvem aspectos subjetivos e culturais. Isto culmina na dificuldade de padronização do conceito e da investigação, e traz a necessidade de criar escalas que consigam se dissociar melhor dos aspectos religiosos, para que a espiritualidade possa ser mais bem aferida. Até o momento, não existe escala padrão ouro para avaliação da espiritualidade $\mathrm{e}^{25,26}$.

A par da dimensão biológica, intelectual, emocional e social, a espiritualidade constitui aquilo que determina a singularidade do indivíduo ${ }^{16}$. E, em última análise, se propõe a buscar o sentido fundamental da existência ${ }^{36}$, estando aí a necessidade do autoconhecimento. E, uma vez que o ser humano visa a sua essência, está indo ao encontro de sua espiritualidade; e quando esta visa ao bem-estar do outro, o indivíduo exerce a ética $^{19}$, lançando mão, portanto, de sua competência moral e desenvolvendo as habilidades e competências no lidar com o outro nos diversos dilemas da vida.

Então, mudanças paulatinas na educação médica para viabilizar profissionais que consigam ir além do fenômeno físico são necessárias, para que os estudantes aprendam e consigam ajuizar de forma mais integral e desenvolvida as situações e vivências profissionais (e da vida) e, portanto, também sejam moralmente mais competentes. 


\section{CONCLUSÕES}

Foi observado que estudantes com menor grau de espiritualidade tiveram tendência a ser moralmente mais competentes. Contudo, existe o viés do aspecto da religiosidade, aspecto mais dogmático, uma vez que no grupo estudado a maioria possui religião (católica), e o questionário de espiritualidade tem uma dimensão relacionada à religiosidade. Este estudo, assim, está de acordo com outros que mostram que a religiosidade dogmática dificulta o desenvolvimento do julgamento e da competência moral.

Outro achado do estudo foi que não houve regressão da competência moral com o avanço da graduação médica, diferindo da maioria dos estudos. Está aí, provavelmente, a importância da educação médica, pois a alta qualidade de educação, com possibilidade de maior tomada de responsabilidade e oportunidade de reflexão com auxílio, leva a efeitos positivos sobre os alunos dogmáticos e não dogmáticos.

São necessários novos instrumentos que consigam aferir o aspecto da espiritualidade, distanciando-o da religiosidade, uma vez que a espiritualidade já é considerada por diversos estudos, instituições e organizações de saúde como um aspecto importante na medicina e na saúde integral. E uma vez que a espiritualidade é a dinâmica de aproximação com o eu profundo (o autoconhecimento), sua avaliação poderá auxiliar o desenvolvimento moral dos estudantes, para a vivência profissional e para a vida.

\section{REFERÊNCIAS}

1. Bataglia PUR, Morais A, Lepre RM. A teoria de Kohlberg sobre o desenvolvimento do raciocínio moral e os instrumentos de avaliação de juízo e competência moral em uso no Brasil. Estudos de Psicologia 2010;15(1):25-32.

2. Murray ME. Moral development and moral education: an overview [Internet]. In: Nucci L. Studies in social and moral development and education: developing fairness and concern for others [Internet]. Chicago: Universityof Illinois; 1995 [última atualização 1 dez 2008]. Disponível em: http: / / tigger.uic.edu/ lnucci/MoralEd/overviewtext.html

3. Koh C. Moral Development and Student Motivation in Moral Education: A Singapore Study. Australian Journal of Education. 2012; 56(1):83-101.

4. Andreata OP. Ética, prazer e religião nas raízes da antiguidade. In: Anais eletrônicos do 10. Congresso de Teologia da PUCPR; 2011; Curitiba, Brasil. Disponível em: http:/ / www.pucpr.br/eventos/congressoteologia/2011/

5. d'Avilla RL. A ética médica e a bioética como requisitos do ser moral: ensinando habilidades humanitárias em medicina. Revista Bioética. 2010;18(2):311-27.
6. Biaggio AMB. Kohlberg e a "Comunidade Justa": promovendo o senso ético e a cidadania na escola. Psicol. Reflex. Crit. 1997; 10(1):47-69.

7. Espíndola MZBL, Lyra VB. O desenvolvimento moral em Lawrence Kohlberg: uma revisão. Humanidades em Foco 2005; 6:3.

8. Bataglia PUR. A validação do Teste de Juízo Moral (MJT) para diferentes culturas: o caso brasileiro. Psicol. Reflex. Crit 2010; 23(1).

9. Crain WC. Theories of Development. Prentice-Hall. $1985 ; 118-136$

10. Lind G. Moral Dilemma Discussion Revisited - The Konstanz Method. 2005; 1(1).

11. Self DJ, Schrader DE, Baldwin DC Jr, Wolinsky FD. The moral development of medical students: a pilot study of the possible influence of medical education. Med Educ. 1993;27(1):26-34.

12. Branch Jr W. Supporting the moral development of medical students. J Gen Intern Med. 2000; 15(7): 503-508.

13. Hébert PC, Meslin EM, Dunn EV. Measuring the ethical sensitivity of medical students: a study at the University of Toronto. J Med Ethics. 1992;18(3):142-7.

14. Lind G. Moral Regression in Medical Students and Their Learning Environment. Revista Brasileira de Educação Médica. 2000;24(3):24-33.

15. Feitosa HN, Rego S, Bataglia PUR, Sancho KFCB, Rego G, Nunes R. Moral judgment competence of medical students: a transcultural study. Adv Health SciEduc Theory Pract. 2013;18(5):1067-85.

16. Pinto C, Pais-Ribeiro JL. Construção de Uma Escala de Avaliação da Espiritualidade em Contextos de Saúde.

ArquiMed. 2007; 21(2):47-53

17. Lucchetti G, Lucchetti ALG, Espinha DCM, Oliveira LR, Leite JR, Koening H. Spirituality and health in the curricula of medical schools in Brazil BMC Medical Education. 2012;12:78.

18. Vasconcelos EM. Espiritualidade na educação popular em saúde. Cad. CEDES [online].2009; 29(79):323-333.

19. Guck TP, Kavan MG. Medical student beliefs: spirituality's relationship to health and place in the medical school curriculum. Med Teach. 2006;28(8):702-7.

20. Anandarajah G, Mitchell MA. Spirituality and medicine elective for senior medical students: 4 years' experience, evaluation, and expansion to the family medicine residency. Fam Med. 2007;39(5):313-5.

21. Dal-farra RA, Geremia C. Educação em saúde e espiritualidade: proposições metodológicas. Rev. bras. educ. med. [online]. 2010;34(4):587-597. 
22. Bataglia PUR. A validação do Teste de Juízo Moral (MJT) para diferentes culturas: o caso brasileiro. Psicol. Reflex. Crit. [online]. 2001; 23(1) [capturado em: 04 dez 2013]; 83-91.

23. Vieira HC, Castro AE, Júnior VFS. O uso de questionários via e-mail em pesquisas acadêmicas sob a ótica dos respondentes. XIIISeminário de administração. 2010.

24. Marconi MA, Lakatos EM. Fundamentos de metodologia científica. 6.ed. São Paulo: Atlas; 2005.

25. Borges DC, Anjos GL, Oliveira LR, Leite JR, Lucchetti G. Saúde, espiritualidade e religiosidade na visão dos estudantes de medicina. Rev Bras Clin Med. São Paulo 2013;11(1):6-11.

26. Lucchetti G, Lucchetti ALG, Valada H. Measuring spirituality and religiosity in clinical research: a systematic review of instruments available in the Portuguese language. Sao Paulo Med J. 2013; 131(2):112-22.

27. Liquat AW. Effect of dogmatic religiosity and educational environment on moral judgment competence. 2012. Dissertação [Mestrado] - Department of Psychology, Faculty of Social Sciences, International Islamic University Islamabad.

28. Saeidi-Parvaneh S. Moral, Education and Religion in Iran: The Significance of Higher Education for the Development of Moral Judgment and Discourse Competence in a Country Shaped by Religion. Constance, Univ., Diss., 2011

29. Bataglia PUR, Schillinger-Agati M, Torres SS, Crivelaro D, Oliveira D, Quevedo T. The development of moral competence and religious commitment in Brazil. Paper presented at the 28th Annual Meeting of the Association for Moral Education, Chicago, U.S.A; 2002.

30. Schillinger-Agati M, Lind G. Moral Judgement Competence in Brazilian and German University Students. Annual Meeting ofthe American EducationResearch Assotiation; 2003.

31. Feitosa HN, Rego S, Bataglia P, Rego G, Nunes R. Competência de Juízo Moral dos Estudantes de Medicina: um Estudo Piloto. Revista Brasileira de Educação Médica 2013; 37(1):5-14.
32. Hren D, Marušić M, Marušić A. Regression of Moral Reasoning during Medical Education: Combined Design Study to Evaluate the Effect of Clinical Study Years. PLoSONE 2011; 6(3): 17406.

33. Patenaude J, Niyonsenga T, Fafard D. Changes in students' moral development during medical school: a cohort study. CMAJ. 2003;168(7):840-4.

34. Faculdade Pernambucana de Saúde [homepage na internet]. Matriz curricular e objetivos da graduação em medicina da Faculdade Pernambucana de Saúde. [acesso em 15 abr 2015]. Disponível em: https://www.fps.edu.br/ cursos/interna/graduacao/medicina

35. Ferreira AGC, Melo WM, Cavalcanti HAF. Grupo de estudos em saúde e espiritualidade: um espaço para discussão na formação acadêmica. In: 52. Congresso Brasileiro de Educação Médica, 2014, Joinville. Anais do 52 Congresso Brasileiro de Educação Médica, 2014, v.1.

36. Filho VPD, Sá FC. Ensino médico e espiritualidade. O mundo da saúde São Paulo 2007; 31(2):273-280.

\section{CONTRIBUIÇÃO DOS AUTORES}

Natália Wolmer de Melo: Autoria

Edvaldo Souza: Orientador

Leopoldo Barbosa: Co-orientador

\section{CONFLITOS DE INTERESSE}

Os autores declaram não haver conflitos de interesse.

\section{ENDEREÇO DE CORRESPONDÊNCIA}

Natália Wolmer de Melo

Av.Bernardo Vieira de Melo 2570, apt 1401

Piedade - Jaboatão dos Guararapes

CEP: 54410-340 PE

E-mail: nataliawolmer@gmail.com> 